\title{
Review of: "Epidemic of youth nicotine addiction? What does the National Youth Tobacco Survey 2017-2019 reveal about high school e-cigarette use in the USA?"
}

\author{
Yen-Wen Chen \\ 1 State University of New York at Stony Brook
}

Potential competing interests: The author(s) declared that no potential competing interests exist.

This study provides data on the prevalence of e-cigarette use and its association with lifetime history of tobacco (and related product) use and dependence of tobacco product among high school students in the USA.

Result $: 1^{\text {st }}$ and $2^{\text {nd }}$ paragraph at page $4-5$

1. The results reveal the association of "used 20+ days of e-cigs (current e-cigs use)" and lifetime tobacco use history. What would the odds ratio be if breaking down the DV groups into "not currently use e-cigs", "used 1-19 days in the past 30 days", "used $20+$ days in the past 30 days"?

2. Is the annual increase in current e-cigs use from 2017 to 2019 significant?

3. The $2^{\text {nd }}$ paragraph at page 5 brought back the results of "current e-cigarette use", while the last sentence of the $1^{\text {st }}$ paragraph ("Past 30-day cigarette smoking was strongly associated with cigarette use history in all years") was a little bit confusing. I would recommend to place results related to current e-cigs use together. In addition, what about the ORs for "past 30-days cigarette smoking" associate with lifetime tobacco use history?

4. What would be the possible explanations behind the annual increase in e-cigs use? Would this associate with the increased availability/accessibility or promotion of e-cigs?

5. Is there sex or geographic difference in the ORs?

Result: dependence section

1. The discussion talked about the current e-cigs use associated with minimal dependence, but what about among the current e-cigs users, 15.5\% used 20+ days in the past month in 2019 (the proportion of this population also increased since 2017). What does the authors' interpretation for this?

Result: first product used section

1. From Table 3, people who tried e-cigs first in 2014 had high percentage (87.1\%) reported use e-cigs only in their lifetime, whereas in 2015 , only $5.7 \%$ who tried e-cigs first reported use e-cigs only in their lifetime. What would the interpretation for this? 
Result: trends in use of cigarettes section

1. Did the argument in the last sentence ("This has occurred without any discernible impact on the slope of declining cigarette and combustible use") have statistical analysis to support? Did the increase of e-cigs use (either current or ever tried) associated (or not associated) with the decline of current cigarette smoker or ever tried combustible product?

2. For the figure, the legend should be placed at the side or at the bottom of the figure. What does the "\%" in $y$-axis refer? Current cigarette smoking rate? This information should be displayed in the figure

3. The discussion talked about that the data did not support the initial trying of e-cigs associated with cigarette use. What about those who continue using e-cigs after the first try?

4. The discussion talked about that the high school students reported high percentage of the cigarettes to be the first tobacco product they used and this attributed to the lifetime cigarette use. However, this argument might be confounded the availability/accessibility of e-cigs in 2014 and 2015. What does the authors opinion about the potential confound(s)?

\section{Discussion:}

1. The discussion concluded that the students' perception toward e-cigs as non-tobacco product. Did "tobacco products" in the dependence questions include e-cigs (e.g., did this information is specified in the questions)? This relates to the next argument the authors made ("this suggests their self-concept is not that of being a tobacco product user"). This argument seems speculative because no data (about the perception of e-cigs as tobacco product or not) in this study supporting it.

2. The authors' arguments and conclusion (the last two paragraphs in the discussion) should take into account the abovementioned points and potential confounds. 